\title{
An Almost General Splitting Theorem For Modal Logic
}

\author{
Marcus Kracht \\ II. Mathematisches Institut \\ Arnimallee 3 \\ 1000 Berlin 33 \\ GERMANY
}

February 6, 2008

\begin{abstract}
Given a normal (multi-)modal logic $\Theta$, a characterization is given of the finitely presentable algebras $\mathcal{A}$ whose logics $L \mathcal{A}$ split the lattice of normal extensions of $\Theta$. This is a substantial generalization of [Rautenberg, 1980; Rautenberg, 1977] in which $\Theta$ is assumed to be weakly transitive and $\mathcal{A}$ to be finite. We also obtain as a direct consequence a result by [Blok, 1978] that for all cycle-free and finite $\mathcal{A} L \mathcal{A}$ splits the lattice of normal extensions of $K$. Although we firmly believe it to be true, we have not been able to prove that if a logic $\Lambda$ splits the lattice of extensions of $\Theta$ then $\Lambda$ is the logic of an algebra finitely presentable over $\Theta$; in this respect our result remains partial.
\end{abstract}

\section{A Introduction}

The concept of a splitting has been very fruitful in modal logic. Although splittings originated in lattice theory they proved to have rather surprising applications to modal logics with no obvious relation to lattice theory. The idea of a splitting in modal logic is this: given a logic $\Theta$ is there a $\operatorname{logic} \Theta / \mathcal{A}$ whose models are all the $\Theta$-models that omit $\mathcal{A}$ in the sense that the variety they generate does not contain $\mathcal{A}$, or, equivalently, does there exists a least logic containing $\Theta$ not having $\mathcal{A}$ as a model? If it exists it is called the splitting of $\Theta$ by $\mathcal{A}$.

Splittings first appeared in pure modal logic in [Fine, 1974a] where they were called frame logics. By showing that the lattice of normal extensions of $S 4$ has $\aleph_{0}$ incomplarable splittings, Fine showed that this lattice has $2^{\aleph_{0}}$ elements. In [Blok, 1978], W. Blok applied splittings to obtain completeness results. He proved that a normal extension of $K$ is complete exactly if it is a splitting logic of $K$ and that the degree of incompleteness is $2^{\aleph_{0}}$ otherwise; and that an algebra splits the lattice of $K$ if and only if it is finite, subdirectly irreducible and cycle-free. The first 
general and theoretic approach to splittings was given in [Rautenberg, 1980], [Rautenberg, 1979] and [Rautenberg, 1977]. He not only showed that many important extensions of $K 4$ are splitting logics of $K 4$, but also gave a way of deciding wether or not $\Theta(P)$ is a splitting logic of $\Theta$. However, the splitting theorem presented there only treats weakly transitive logics. In modal logics this is not a severe restriction, but as soon as the language has more than one modal operator-e.g. in tense logics - transitivity is no longer a desirable property.

Our main aim is a general characterization of all algebras which split $\Theta$ for an arbitrary normal modal logic without any assumptions of transitivity. We allow the language to have more than one modal operator, and also the splitting algebra to be infinite. However, we have to assume that the algebras we are dealing with are extit finitely presentable over $\Theta$. The road to the main splitting theorem presented here is quite long compared to the splitting theorem for transitive logics because it is no longer true that only the finitely presentable, subdirectly irreducible (s.i.) algebras induce splittings. Moreover, since $\Theta$ does not necessarily have the finite model property, it is not true that the class of splitting algebras of $\Theta$ coincides with the class of finite s.i. algebras, which is then also the class of finitely presentable s.i. algebras. The investigation into this problem leads directly back to the lattice-theoretic definition of a splitting and the characterization of splitting elements in a lattice.

The first section contains all the relevant lattice facts about splittings. In the next section we introduce modal logics and a new semantics which unifies both modal and dynamic logics. Although we do not include any application for dynamic logic it will become clear that this semantics makes the application of our results even within modal logics easier and more concise. We have tried to keep this part as short as possible. The third part is the heart of the paper, where we prove the splitting theorem. There we try to flesh out the interconnection between the structure of $\Theta-$ algebras and the structure of the lattice of normal extensions of $\Theta$. As a byproduct we get several theorems which are of interest in their own right. They deal with the problem of how to decide whether a s.i. algebra is in the variety generated by a set of other algebras. Finally, a nontrivial example of an infinite splitting algebra is given.

I am very grateful to Prof. Rautenberg for his supervision of my diploma thesis out of which the present paper developed as well as to Frank Wolter for carefully reading this paper and making many helpful suggestions. Some anonymous referees have also helped to significantly improve earlier versions.

\section{B Splittings in Lattices}

The notion of a splitting was first used in the context of varieties of algebras by [McKenzie, 1972]. However, splittings as a concept of lattice theory date back to [Whitman, 1943] who also coined this expression. ${ }^{1}$

\footnotetext{
${ }^{1}$ Whitman claims that [Birkhoff, 1937] uses 'cleavage' in much the same sense as 'splittings' but this is not correct. A cleavage for a prime ideal in a lattice is defined in [Birkhoff, 1937] as a prime quotient $b / a$ such that $a \in P$ but $b \notin P$. Incidentally, our usage of 'splitting' which we borrowed from [McKenzie, 1972] differs sightly from the original one in [Whitman, 1943].
} 
Let $\mathcal{L}:=\langle L, \sqcap, \sqcup, \sqcap, \sqcup, 0,1\rangle$ be a complete lattice. For $a \in L$ define $\mathcal{F} a:=\{x \in L: x \geq a\}$, the filter generated by $a$, and $\mathcal{I} a:=\{x \in L: x \leq a\}$, the ideal generated by $a$. Also define $\mathcal{E} a:=\langle\mathcal{F} a, \sqcap, \sqcup, \sqcap, \sqcup, a, 1\rangle . \mathcal{E} a$ is called the extension lattice of $a$.

Definition $1\langle p, q\rangle$ is called a splitting of $\mathcal{L}$ if $L=\mathcal{F} q+\mathcal{I} p$ that is, $L=\mathcal{F} q \cup \mathcal{I} p$ and $\varnothing=\mathcal{F} q \cap \mathcal{I} p$. In that case we say that $p$ splits $\mathcal{L}$ and that $q$ is its splitting companion. $q$ is uniquely determined by $p$ and denoted by $\mathcal{L} / p$ or sometimes by $0 / p$.

Dually, we say that $q$ co-splits $\mathcal{L}$ if for some $p \mathcal{I} p+\mathcal{F} q=L$. Again, $p$ is uniquely determined by $q$ and called the co-companion of $q$. Hence there is a bijection between splitting elements and co-splitting elements of $\mathcal{L}$. If $p$ splits $\mathcal{L}$ and $p \in \mathcal{F} a$ then $p$ splits $\mathcal{E} a$ and $a / p=a \sqcup 0 / p$. If $p \notin \mathcal{E} a$ we define $a / p:=a$. Splittings can be iterated, e.g. $(\mathcal{L} / p) / r$. There it need not be the case that $r$ splits $\mathcal{L}$; but if it does it is easily checked that $(\mathcal{L} / p) / r=(\mathcal{L} / r) / p=\mathcal{L} / p \sqcup \mathcal{L} / r$. Thus for a set $N$ of splitting elements we define $\mathcal{L} / N:=\sqcup\langle\mathcal{L} / p: p \in N\rangle$.

Definition $2 a \in L$ is called irreducible if $a=\prod\langle x(i): i \in I\rangle$ implies $a=x(i)$ for some $i \in I$. $a \in L$ is called prime if $a \geq \Pi\langle x(i): i \in I\rangle$ implies $a \geq x(i)$ for some $i \in I$.

The dual notions are join-irreducible and join-prime. If $a$ is prime $a$ is also irreducible. If the lattice is upper continuous, i.e. if $a \sqcap \bigsqcup\left\langle b_{i}: i \in I\right\rangle=\bigsqcup\left\langle a \sqcap b_{i}: i \in I\right\rangle$, then every join-irreducible element is also join-prime. This is the case with the lattice of normal extensions of a modal logic.

Theorem 3 (McKenzie) $p$ splits $\mathcal{L}$ iff $p$ is prime in $\mathcal{L}$.

By duality, $q$ co-splits $\mathcal{L}$ iff $q$ is join-prime in $\mathcal{L}$. Thus we have a bijection $\sigma: \Sigma^{*} \longrightarrow \Sigma_{*}$ from the set of prime elements $\Sigma^{*}$ of $\mathcal{L}$ onto the set $\Sigma_{*}$ of join-prime elements of $\mathcal{L}$ defined by $\sigma(p):=\mathcal{L} / p$. Moreover, if $p \leq q$ in $\Sigma^{*}$ then $\sigma(p) \leq \sigma(q)$ in $\Sigma_{*}$ and vice versa, in other words, $\sigma$ is an isomorphism of posets.

\section{Modal Logics: Syntax and Semantics}

We assume that the reader is familiar with the basic ideas of modal logics and elementary dynamic logics. For the latter we refer to Harel [7]. For several reasons we use the language of dynamic logics. First of all they are more general and flexible than modal logics. Second, the development of dynamic logic cannot be ignored by modal logicians. Instead, they should make their results accessible to a wider audience by adopting the new language of programs. And third, most of our general results can be stated in a more compact way in terms of programs. Thus our language is the language of EPDL (Elementary Propositional Dynamic Logic). It consists of a countable set Var of proposition variables, a countable set $\operatorname{Prg}:=\left\{\pi_{i}: i \in \omega\right\}$ of elementary program constants, the connectives $\neg, \wedge, \vee, \rightarrow, \leftrightarrow, ;, \cup$ as well as [ ] and $<>$. We also use the symbols 0 (false), 1 
(true) and 1 (no operation). The brackets [ ] and $<>$ turn programs into modal operators. Thus if $\mu$ is a program and $P$ a proposition then $[\mu] P$ and $\langle\mu\rangle P$ are propositions. By $\mathcal{L}$ we denote the set of propositions generated by $\operatorname{Var}$ and $\operatorname{Prg}$, by $\mathcal{L}_{n}$ the subset of propositions generated by $\operatorname{Var}$ and $\left\{\pi_{i}: i \in n\right\}$. However, it should be said that none of our results ultimately depends on the choice of the language of programs as long as it contains ' $U$ ' since all our results only depend on the semilattice-structure of the programs defined by ' $U$ '. It is only for shortness and simplicity that we have not included the star ' $\star$ '. Or we could have added the intersection ' $\cap$ ' or negation ' $~$ ' of programs (which for a program $\pi$ yields a program $\backslash \pi$ which makes all and only those worlds accessible which are inaccessible for $\pi$ ). The splitting theorem and all the others remain valid. However, our results do not extend to dynamic logics which have the '?' because we have no semantics for it. In fact, the semantic framework used here does not extend to any semantics for '?'.

The important innovation of dynamic logic is the notion of a program as an object of a different category. In EPDL and PDL the programs form an algebra generated by a set of basic programs in $\operatorname{Prg}$ and $;, \cup$ and (in PDL) ${ }^{\star}$. We call this algebra a $P$-ring:

Definition 4 An algebra $X=\langle X, 1, \cup, ;\rangle$ of type $\langle 0,2,2\rangle$ is called $P$-ring iff the following equations hold:

$$
\begin{array}{ll}
(r a ;) & \pi ;(\sigma ; \tau)=(\pi ; \sigma) ; \tau \\
(r 1 ;) & \pi ; 1=1 ; \pi=\pi \\
(r a \cup) & \pi \cup(\sigma \cup \tau)=(\pi \cup \sigma) \cup \tau \\
(r c \cup) & \pi \cup \sigma=\sigma \cup \pi \\
(r i \cup) & \pi \cup \pi=\pi \\
(r d l) & \pi ;(\sigma \cup \tau)=\pi ; \sigma \cup \pi ; \tau \\
(r d r) & (\pi \cup \sigma) ; \tau=\pi ; \tau \cup \sigma ; \tau
\end{array}
$$

A P-ring is always assumed to be finitely generated by $X_{0} \subset P r g$.

Definition 5 An algebra $\mathcal{X}=\left\langle X, 1, \cup, ;,{ }^{\star}\right\rangle$ of type $\langle 0,2,2,1\rangle$ is called a $P^{\star}$-ring if $\mathcal{X} \uparrow\{1, \cup, ;\}$ is a P-ring and for all $\pi \in X$

$$
\left(i n^{*}\right) \quad(1 \cup \pi) ; \pi^{*}=\pi^{*}
$$

This equation is reflected in dynamic algebras by the induction axiom

$$
\left(r c^{*}\right) \quad\left[\pi^{*}\right](p \rightarrow[\pi] p) . \rightarrow . p \rightarrow\left[\pi^{*}\right] p .
$$

As we said already, we will not discuss the case of the star (viz. dynamic algebras) for this would make our introduction unnecessarily long. However, it should be clear that it runs exactly parallel.

Definition 6 Let $\boldsymbol{A}=\langle A, 1, \backslash, \cap\rangle$ and $\boldsymbol{B}=\langle B, 1, \backslash, \cap\rangle$ be boolean algebras. $\tau: \boldsymbol{A} \longrightarrow \boldsymbol{B}$ is called a hemimorphism, if $\tau 1=1$ and $\tau(a \cap b)=\tau a \cap \tau b$ for all $a, b \in A$. The set of all hemimorphisms 
from $\boldsymbol{A}$ to $\boldsymbol{B}$ is denoted by $\operatorname{Hem}(\boldsymbol{A}, \boldsymbol{B})$. If $\boldsymbol{A}=\boldsymbol{B}$ we simplify this to $\operatorname{Hem}(\boldsymbol{A})$. If $\sigma, \tau \in \operatorname{Hem}(\boldsymbol{A}, \boldsymbol{B})$ we define $\sigma \cup \tau$ by $(\sigma \cup \tau) a:=\sigma a \cap \tau a$.

Proposition $7 \mathcal{H}(\boldsymbol{A}):=\langle\operatorname{Hem}(\boldsymbol{A}), i d, \cup, \circ\rangle$ is a $P$ - ring.

Definition 8 Let $\mathcal{X}$ be a $P$-ring. $A \mathcal{X}$-algebra is a pair $\mathcal{A}=\langle\boldsymbol{A}, f\rangle$ where $\boldsymbol{A}$ is a boolean algebra and $f: \mathcal{X} \longrightarrow \mathcal{H}(\boldsymbol{A})$ a P-homomorphism. ${ }^{2}$ A homomorphism between $\mathcal{X}$-algebras $\langle\boldsymbol{A}, f\rangle$ and $\langle\boldsymbol{B}, g\rangle$ is a pair $\langle h, t\rangle$ of mappings, $h \in \operatorname{Hom}(\boldsymbol{A}, \boldsymbol{B})$ and $t: i m(f) \longrightarrow i m(g)$ a P-homomorphism, such that for all $\pi \in X$ :

$(x h) \quad h \circ f(\pi)=g(\pi) \circ h$

(xp) $\quad t(f(\pi))=g(\pi)$
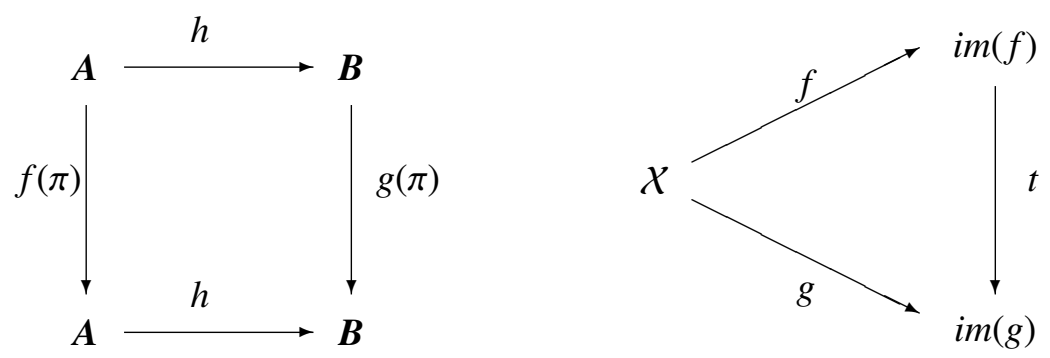

The idea of this semantics is best explained with an example. Take the variety $\mathcal{V}_{n}$ of algebras with $n$ modal operators. $\mathcal{A}=\left\langle\mathbf{A},\left\langle\tau_{i}: i \in n\right\rangle\right\rangle \in \mathcal{V}_{n}$ is then a boolean algebra with $n$ operators. Alternatively, if $\mathcal{X}_{n}$ is the P-ring freely generated by $\left\{\pi_{i}: i \in n\right\}$, the assignment $f: \pi_{i} \mapsto \tau_{i}$ induces a P-homomorphism $f: \mathcal{X}_{n} \longrightarrow \operatorname{Hem}(\mathbf{A})$ whose direct image is the subring generated by the $\tau_{i}$. This shows how to turn an n-modal algebra into a $\mathcal{X}_{n}$-algebra.

Under certain circumstances it is possible to replace $\mathcal{X}_{n}$ by a homomorphic image. For consider the case when $f: \mathcal{X}_{n} \longrightarrow \operatorname{Hem}(\mathbf{A})$ factors through $g: \mathcal{X}_{n} \longrightarrow \mathcal{Y}$. Then $\langle\mathbf{A}, f\rangle$ can equally be viewed as a $\mathcal{Y}$-algebra. The conditions under which this identification is possible can be spelled out explicitly. For $f$ factors through $g$ iff $f(\pi)=f(\sigma)$ for all $\pi, \sigma$ such that $g(\pi)=g(\sigma)$. So we must have $f(\pi)(a)=f(\sigma)(a)$ for all $a \in A$ which is equivalent to $\langle\mathbf{A}, f\rangle \vDash[\pi] p \leftrightarrow[\sigma] p$ for all such pairs. The algebras satisfying these identities therefore form a variety. A particular example is the class of interior algebras or S4-algebras, which are modal algebras satisfying the identities $a \cap \square a=\square a$ and $\square \square a=\square a$. An interior algebra as an $\mathcal{X}_{1}$-algebra can also be construed as a $\mathcal{X}_{i}$-algebra, where $\mathcal{X}_{i}$ is $\mathcal{X}_{1}$ factored through $1 \cup \pi_{0}=\pi_{0}$ and $\pi_{0} ; \pi_{0}=\pi_{0}$. It turns out that $\mathcal{X}_{i}$ has only two elements, namely 1 and $\pi_{0}$. In general, distinct logics need not have dictinct rings of programs. The interior algebras provide an example. It is readily checked that if an extension $\Lambda$ of S4 has a proper image of $\mathcal{X}_{i}$ as it's ring programs, this ring must satisfy $1=\pi_{0}$. Consequently, $\Lambda \supseteq S 4(p \leftrightarrow \square p)$.

\footnotetext{
${ }^{2}$ We generally refer to $\mathcal{X}$-algebras as modal or multimodal algebras. If $\mathcal{X}$ is a $P^{*}$-ring we call them $d y n a m i c$ algebras.
} 
The kernel of a homomorphism of $\mathcal{X}$-algebras is an open filter: a subset $F \subseteq A$ is called an open filter if it is closed under

$$
\begin{aligned}
& (f \leq) \quad a \in F \text { and } a \leq b \Rightarrow b \in F \\
& (f \cap) \quad a, b \in F \Rightarrow a \cap b \in F \\
& (f \square) \quad a \in F \text { and } \pi \in X \Rightarrow[\pi] a \in F
\end{aligned}
$$

Open filters are in one-to-one correspondence to surjective homomorphisms.

In the same way we define $\mathcal{X}$-frames (or Kripke-frames) as we have done for $\mathcal{X}$-algebras. A $\mathcal{X}$ frame $\Gamma$ is a pair $\langle G, \gamma\rangle$ where $G$ is a set (the set of worlds) and $\gamma: \mathcal{X} \longrightarrow \operatorname{Rel}(G)$ a homomorphism into the $P$-ring of relations over $G$. We leave the details of this construction to the reader. Mappings between $\mathcal{X}$-frames are called p-morphisms. As an example he may check the definitions in the S4case which is the most simple one. Other $P$-rings of interest are $\mathcal{X}_{n}$, the $P$-ring freely generated by $n$ elements, and $\mathcal{X}_{n}^{k}$, the $P$-ring generated by $n$ elements in which every program is $k$-transitive. To define $\mathcal{X}_{n}^{k}$ we need some notation:

$$
\begin{array}{lll}
(\text { exm }) & \pi^{0}:=1 & \pi^{m+1}:=\pi^{m} ; \pi \\
(\text { ex }) & \pi^{(0)}:=1 & \pi^{(m+1)}:=\pi^{(m)} \cup \pi^{m+1} \\
(d f \xi) & \xi:=\bigcup\left\langle\pi_{i}: i \in n\right\rangle &
\end{array}
$$

$\mathcal{X}_{n}^{k}$ is generated by $\left\{\pi_{i}: i \in n\right\}$ and the equation $\xi^{(k+1)}=\xi^{(k)} . \mathcal{X}_{n}^{k}$ is called $k$-transitive. A logic $\Theta$ is called $k$-transitive if $\left[\xi^{(k+1)}\right] p \leftrightarrow\left[\xi^{(k)}\right] p \in \Theta$ and weakly transitive if it is $k$-transitive for some $k \in \omega$. In a frame for a $k$-transitive logic every path consisting of more than $k$ successive moves from one point to another can be replaced by a path of at most $k$ moves joining these points.

Now let $\mathcal{X}$ be any $P$-ring generated by $\left\{\pi_{i}: i \in n\right\}, Y \subseteq \operatorname{Var}$ a set of proposition variables. $\mathcal{F}(Y):=\left\langle\operatorname{Fr}_{X}(Y), p\right\rangle$ denotes the free $\mathcal{X}$-algebra generated by $Y . \operatorname{Fr}_{X}(Y)$ is the boolean algebra generated by $X$ and $Y$ and the equations

$$
\begin{array}{ll}
(i e ;) & {[\mu ; \sigma] p=[\sigma]([\mu] p)} \\
(i e \cup) & {[\mu \cup \sigma] p=[\mu] p \wedge[\sigma] p} \\
(i e 1) & {[1] p=p} \\
(i e \mu) & {[\mu] 1=1} \\
(\operatorname{sm} \wedge) & {[\mu](p \wedge q)=[\mu] p . \wedge .[\mu] q \text { for all } \mu, \sigma \in X}
\end{array}
$$

$p: \mathcal{X} \longrightarrow \mathcal{H}\left(\operatorname{Fr}_{\mathcal{X}}(Y)\right)$ is defined by $p(\pi)(Q):=[\pi] Q$. If $\mathcal{A}=\langle\mathbf{A}, f\rangle$ is a $\mathcal{X}$-algebra and $\beta: Y \longrightarrow A$ a valuation then $\beta$ defines a unique extension $h_{\beta}:\langle\bar{\beta}, t\rangle:\left\langle\operatorname{Fr}_{\mathcal{X}}(Y), p\right\rangle \longrightarrow\langle\mathbf{A}, f\rangle$. We say, $\langle\mathcal{A}, \beta, a\rangle$ is a model for $P$ if $0<a \leq \bar{\beta}(P)$ and we write $\mathcal{A}, \beta \models P>0$. We say, $\langle\mathcal{A}, \beta\rangle$ satisfies $P$ if $\langle\mathcal{A}, \beta, 1\rangle$ is a model for $P$ and write $\mathcal{A}, \beta \models P$. This corresponds to the usual definition of a model where instead of an algebra a frame $g$ is given: $\langle g, \beta, s\rangle$ is a model for $P$ iff $g, \beta, s \models P$. We also say that $\langle g, \beta\rangle$ is a model for $P$ if such an $s \in g$ exists. The logic $L \mathcal{A}$ of $\mathcal{A}$ is the set of all $P \in \mathcal{L}_{n}$ which are always satisfied: $L \mathcal{A}:=\left\{P \in \mathcal{L}_{n}: \forall \beta: \operatorname{var}(P) \longrightarrow A: \mathcal{A}, \beta \models P\right\}$. 
A normal ( $n$-)modal logic is a subset of $\mathcal{L}_{n}$ which contains the axioms of classical logic, the axioms corresponding to the above equations,

$$
(b d) \quad \vdash[\pi](p \rightarrow q) . \rightarrow .[\pi] p \rightarrow[\pi] q, \quad \pi \in X
$$

and which is closed under substitution of formulas for proposition variables, modus ponens and necessitation: $P /[\pi] P, \pi \in X$. Usually, we denote logics by upper case Greek letters. A logic $\Theta$ corresponds to a uniquely determined variety $\mathcal{V}(\Theta):=\{\mathcal{A}: L \mathcal{A} \supseteq \Theta\}$ of $\mathcal{X}_{n}$-algebras and every variety of $\mathcal{X}_{n}$-algebras $V$ determines a logic $\mathcal{L}(V):=\bigcap\langle L \mathcal{A}: \mathcal{A} \in V\rangle$. The minimal normal n-modal logic is denoted by $K_{n}$.

\section{Splitting Varieties of Modal Logics}

\section{D.1 Irreducible and Prime Logics}

The aim of this section is to describe how the concepts from lattice theory are translated into properties of algebras and varieties of modal algebras. We assume that the language is some fixed $\mathcal{L}_{n}$ and the logic $\Theta \subseteq \mathcal{L}_{n}$. We will show that the splitting logics are of type $L \mathcal{A}$ where $\mathcal{A}$ is not only subdirectly irreducible (henceforth s.i.) but also prime in $\mathcal{V}(\Theta)$, and then proceed to describe the notion of primeness in terms of algebras and their varieties. We have seen in section A that in contrast to irreducibility of an element $a$ in a lattice $\mathcal{L}$, which depends only on the structure of the local extension lattice $\mathcal{E} a$, primeness depends on the overall structure of $\mathcal{L}$. It is therefore natural to expect that irreducibility corresponds to an internal property of an algebra i.e. a property that depends only on the structure of the algebra itself; and that primeness of an algebra is dependent also on the variety it is contained in. But even in the case of irreducibility the matter is quite complicated. Suppose we are given a $P$-ring $\mathcal{X}$ and the corresponding variety $\mathcal{V}$ of $\mathcal{X}$-algebras and $\Theta$ correponds to a subvariety of $\mathcal{V}$. Then we have the

Theorem $9 \Lambda \in \mathcal{E} \Theta$ is irreducible only if $\Lambda=L \mathcal{A}$ for a subdirect irreducible $\mathcal{A}$.

Proof: Surely we have $\Lambda=L \mathcal{A}$ for some $\mathcal{A} \in \mathcal{V}$. If $\mathcal{A}$ is not s.i., say $\mathcal{A}$ is a subdirect product of $\left\langle\mathcal{A}_{i}: i \in I\right\rangle$, where every $\mathcal{A}_{i}$ is s.i., then we have $L \mathcal{A}=\bigcap\left\langle L \mathcal{A}_{i}: i \in I\right\rangle$. Hence there is a $\mathcal{A}_{i}$ such that $L \mathcal{A}_{i}=L \mathcal{A}=\Lambda$.

A criterion for subdirect irreducibility is given in [Rautenberg, 1980]:

Theorem $10 \mathcal{A} \in \mathcal{V}$ is s.i. iff there is a $c \in A \backslash\{1\}$ such that $\forall a \in A \backslash\{1\}: \exists \mu \in X:[\mu] a \leq c$. Every $c$ that has this property is called an opremum of $\mathcal{A}$. $c$ is not uniquely determined. If $\mathcal{A}$ is finite then $\mathcal{A}$ is s. i. iff the corresponding frame is generated by a single point.

But the converse of Theorem 9 is generally false. As an example take the S4-frame $\langle\{\infty\} \cup \omega, \triangleleft\rangle$ with $s \triangleleft t$ iff $s=\infty$ or $s \geq t$. This frame is generated by $\infty$ and therefore the algebra $\mathcal{A}$ of finite 
and cofinite subsets of that frame is s.i. However, it can be shown that $L \mathcal{A}=S 4 . G r z .3$ which has the finite model property (f.m.p.) and is therefore not irreducible in $\mathcal{E} K$. To obtain a converse of Theorem 9 we remind ourselves of the fact that in a lattice an element $a$ is irreducible iff it is prime in $\mathcal{E} a$. So we find

Theorem $11 \Lambda$ is irreducible in $\mathcal{E} \Theta$ if and only if $\Lambda=L \mathcal{A}$ for a s.i. algebra such that $L \mathcal{A}$ is prime in $\mathcal{E} L \mathcal{A}$.

which reduces the problem of finding the algebras whose logics are irreducible to the more general problem of characterizing splitting algebras. Therefore we will now attempt such a characterization.

By analogy, an algebra $\mathcal{A}$ or a frame $\Gamma$ is called prime in $\mathcal{E} \Theta$ or $\mathcal{V}(\Theta)$ if $L \mathcal{A}(L \Gamma)$ is prime in $\mathcal{E} \Theta$. In previous work only finite algebras have been considered (see for example [Blok, 1978], [Rautenberg, 1980; Rautenberg, 1977]). This restriction is only justified if the base logic $\Theta$ has f.m.p. For if a logic is prime in $\mathcal{E} \Theta$ it is also irreducible and so it is generated by a s.i. algebra. Thus, if $\Theta$ has f.m.p. then only finite algebras can induce splittings of $\mathcal{E} \Theta$. Since we want the most general characterization of splitting algebras we do not want to make any assumptions on $\Theta$. However, we have not been able to obtain full generality. Our characterization is restricted to the class of finitely presentable algebras over $\Theta$, which, from a technical point of view, is the most natural class of algebras to work with.

\section{D.2 Finetely Presentable Algebras}

Definition 12 Let $\mathcal{A} \in \mathcal{V}(\Theta)$ be finitely generated by $\left\{a_{i}: i \in k\right\}$. $\mathcal{A}$ is called finitely presentable over $\Theta$ if there exists a formula $\Delta \in \mathcal{L}_{n}$ so that $\operatorname{var}(\Delta) \subseteq\left\{p_{i}: i \in k\right\}$ and the canonical homomorphism $p r: \mathcal{F}_{\Theta}(\operatorname{var}(\Delta)) /\langle\Delta\rangle \longrightarrow \mathcal{A}$ defined by $\operatorname{pr}\left(p_{i}\right)=a_{i}$ is an isomorphism. $\mathcal{F}_{\Theta}(Y)$ denotes the free $\Theta$-algebra generated by $Y$ and $\langle\Delta\rangle$ the open filter generated by $\Delta$. We call $\Delta$ a diagram of $\mathcal{A}$ over $\Theta$. $\Delta$ is not uniquely determined.

We also say that $\mathcal{A}$ is k-presentable to indicate that $\Delta$ is based on no more than $k$ variables. Similarly we use the expression that $\mathcal{A}$ is $k$-generated if a set of no more than $k$ elements of $A$ exists which generates $\mathcal{A}$.

Example: Let $\mathcal{R}$ denote the recession frame, which is defined by $\mathcal{R}:=\langle\omega, \triangleleft\rangle$ where $n \triangleleft m$ iff $n \leq m+1$ (see [Blok, 1978] and [Rautenberg, 1977]). Let $\mathcal{B}$ be the algebra of the finite and cofinite subsets of $\omega$ and $\Theta:=L \mathcal{B}=K(\square \diamond p \rightarrow \diamond \square \square p, \square p \rightarrow p, \diamond p \wedge \square(p \rightarrow \square p) \rightarrow p)$. $\mathcal{B}$ is 1-generated e.g. by $\{0\} \subseteq \omega$. Thus we have a surjective homomorphism $\pi: \mathcal{F}(p) \longrightarrow \mathcal{B}$ defined by $\pi(p)=\{0\}$. $\{0\}$ satisfies the equation $\square\{0\}=\emptyset$ and if we let $\Delta:=\neg \square p$ we get $\mathcal{B} \cong \mathcal{F}_{\Theta}(p) /\langle\Delta\rangle$. It is known (see [Blok, 1980], Theorem 5.2) that $\mathcal{B}$ is s.i. and $\mathcal{E} \Lambda \cong 3$ (see picture). Hence $\mathcal{B}$ is prime in $\mathcal{E} \Theta$ and induces a splitting $\mathcal{E} \Theta / \Theta=L \bullet \bullet$, where $\bullet$ denotes the frame with one reflexive point. 


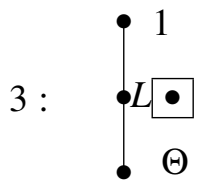

If $\mathcal{A}$ is finite then $\mathcal{A}$ is k-presentable iff it is k-generated. In particular, $\mathcal{A}$ is k-presentable for $k=\operatorname{card}(A)$ :

Proposition 13 Let $\mathcal{A}$ be finite. Define

$$
\begin{aligned}
\Delta(\mathcal{A}):= & \wedge\left\langle p_{a} \vee p_{b} \leftrightarrow p_{a \cup b}: a, b \in A\right\rangle \\
& \wedge \bigwedge\left\langle\neg p_{a} \leftrightarrow p_{\backslash a}: a \in A\right\rangle \\
& \wedge \bigwedge\left\langle[\pi] p_{a} \leftrightarrow p_{[\pi] a}: a \in A, \pi \in X_{0}\right\rangle
\end{aligned}
$$

Then $\Delta(\mathcal{A})$ is a diagram for $\mathcal{A}$ over $\Theta$.

Proof: Consider $\epsilon: \mathcal{F}_{\Theta}(\operatorname{var}(\Delta)) \longrightarrow \mathcal{A}: p_{a} \mapsto a . \epsilon$ is surjective and factors through $p r$, that is, $\epsilon=p r \circ \kappa$ for a homomorphism $\kappa$. Since $p r \circ \kappa=\epsilon$ is surjective, $p r$ is surjective. It remains to show that $p r$ is injective. This is done by showing that $\kappa(P)=\kappa(Q)$ iff $P \leftrightarrow Q \in\langle\Delta\rangle$ and for every $P$ there is a $a \in A$ such that $P \leftrightarrow p_{a} \in\langle\Delta\rangle$ which is proved by induction on $P$. Hence $\operatorname{card}(\operatorname{im}(\kappa))=\operatorname{card}(A)$ and since $A$ is finite, $p r$ is injective.

\section{D.3 Varieties of Modal Algebras}

Definition 14 Let $\Delta$ be a diagram of $\mathcal{A}$ over $\Theta, \mathcal{B} \in \mathcal{V}(\Theta)$ and $\mu \in X$. B is said to be $\mu$-consistent with $\mathcal{A}$, if a valuation $\beta: \operatorname{var}(\Delta) \longrightarrow B$ exists such that $\mathcal{B}, \beta \models \neg p_{c} \wedge[\mu] \Delta>0$, where $p_{c} \in \operatorname{var}(\Delta)$, c being an opremum of $\mathcal{A}$. If $M \subseteq X$ is a set of programs, $\mathcal{B}$ is called $M$-consistent with $\mathcal{A}$ if a valuation $\beta: \operatorname{var}(\Delta) \longrightarrow B$ exists satisfying $\mathcal{B}, \beta \models \neg p_{c} \wedge[\mu] \Delta>0$ for all $\mu \in M$.

We say that $M$ exhausts $\mathcal{X}$ if $\forall \xi \in X \exists \mu \in M: \xi \subset \mu$. If $\mathcal{B}$ is $\mu$-consistent with $\mathcal{A}$ for every $\mu \in M$ (M-consistent with $\mathcal{A})$ and $M$ exhausts $\mathcal{X}$ then $\mathcal{B}$ is said to be weakly consistent with $\mathcal{A}$ (extremely consistent with $\mathcal{A}$ ).

Remark: $\mathcal{B}$ is $\mu$-consistent with $\mathcal{A}$ iff $\exists \beta: \mathcal{B}, \beta \vDash[\mu] \Delta \wedge \neg p_{c}>0$ iff $[\mu] \Delta \rightarrow p_{c} \notin L \mathcal{A}$ iff $\operatorname{Con}_{L \mathcal{A}}[\mu] \Delta \rightarrow p_{c}$. Also, $\mathcal{B}$ is $M$-consistent with $\mathcal{A}$ iff $\operatorname{Con}_{L \mathcal{A}}\left\{[\mu] \Delta \rightarrow p_{c}: \mu \in M\right\}$. If $M$ exhausts $X$ this is equivalent to $\operatorname{Con}_{L \mathcal{A}}\left\{[\pi] \Delta \rightarrow p_{c}: \pi \in X\right\}$. Hence extreme consistency does not depend on $M$. Weak consistency does also not depend on the choice of $M$.

Theorem 15 Let $\mathcal{A}$ be s.i. and finitely presentable over $\Theta$. Then the following assertions are equivalent for all $\mathcal{B} \in \mathcal{V}(\Theta)$ : 
(i) $\mathcal{B}$ is extremely consistent with $\mathcal{A}$

(ii) $\mathcal{A} \in \boldsymbol{S H}(\mathcal{B})$

(iii) $\mathcal{A} \in \boldsymbol{H} \boldsymbol{S}(\mathcal{B})$

Proof: Let $\Delta$ be a diagram of $\mathcal{A}$ over $\Theta$.

(i) $\Rightarrow$ (ii) : Let $M$ exhaust $\mathcal{X}$ and let $\beta: \operatorname{var}(\Delta) \rightarrow B$ be such that $\mathcal{B}, \beta \models \neg p_{c} \wedge[\mu] \Delta>0$ for all $\mu \in X$. Define $F:=\{b \in B: \exists \mu \in M: \bar{\beta}([\mu] \Delta) \leq b\}$. $F$ is an open filter:

$(f \leq)$ is obviously fulfilled.

( $f \cap$ Let $b_{1}, b_{2} \in F$ and $\bar{\beta}\left(\left[\mu_{i}\right] \Delta\right) \leq b_{i}, i=1,2$. Then there is a $\mu_{3} \supset \mu_{1} \cup \mu_{2}$ for $M$ exhausts $\mathcal{X}$. Hence $\bar{\beta}\left(\left[\mu_{3}\right] \Delta\right) \leq \bar{\beta}\left(\left[\mu_{1} \cup \mu_{2}\right] \Delta\right) \leq \bar{\beta}\left(\left[\mu_{1}\right] \Delta \cap \bar{\beta}\left(\left[\mu_{2}\right] \Delta\right) \leq b_{1} \cap b_{2}\right.$ and therefore $b_{1} \cap b_{2} \in F$.

( $f \square$ ) Let $b \in F$ and $\pi \in X$. We have $\bar{\beta}([\mu] \Delta) \leq b$ for a certain $\mu \in M$ and there is a $\widehat{\mu} \in M$ such that $\mu ; \pi \subset \widehat{\mu}$ and $\bar{\beta}([\widehat{\mu}] \Delta) \leq \bar{\beta}([\mu ; \pi] \Delta)=[\pi] \bar{\beta}([\mu] \Delta) \leq[\pi] b$ whence $[\pi] b \in F$.

Consider the induced mapping $\epsilon: \mathcal{B} \longrightarrow \mathcal{B} / F=: C$ and set $\gamma:=\epsilon \circ \beta: \operatorname{var}(\Delta) \longrightarrow C$. Then $\bar{\gamma}([\mu] \Delta)=1$ for all $\mu \in M$. The morphism $\bar{\gamma}$ factors through $\eta: \mathcal{F}_{\Theta}(\operatorname{var}(\Delta)) \longrightarrow \mathcal{A}$, for $\mathcal{A} \cong$ $\mathcal{F}_{\Theta}(\operatorname{var}(\Delta)) /\langle\Delta\rangle$.

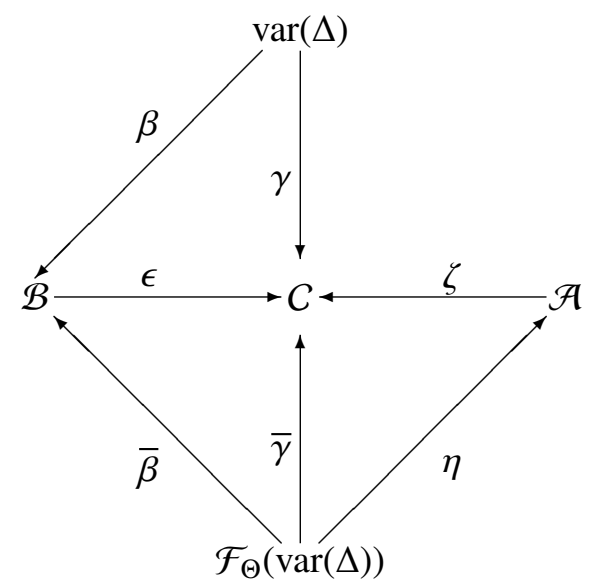

The induced mapping $\zeta$ is injective because $\mathcal{A}$ is s.i. and has a minimal nontrivial congruence relation which is generated by $c$. It is therefore sufficient to show that $\zeta(c) \neq 1$ or equivalently $\underline{\zeta}(\backslash c) \neq 0$. But $\zeta(\backslash c)=\zeta \circ \eta\left(\neg p_{c}\right)=\bar{\gamma}\left(\neg p_{c}\right)=\epsilon \circ \bar{\beta}\left(\neg p_{c}\right)>0$ because for every $a \in F: \bar{\beta}\left(\neg p_{c}\right) \cap a \geq$ $\bar{\beta}\left(\neg p_{c}\right) \cap \bar{\beta}([\mu] \Delta)=\bar{\beta}\left(\neg p_{c} \wedge[\mu] \Delta\right) \neq 0$ for some $\mu \in M$ with $\bar{\beta}([\mu] \Delta) \leq a$. Hence $\zeta$ is injective and $\mathcal{A} \in \mathbf{S}(C) \subseteq \mathbf{S H}(\mathcal{B})$.

(ii) $\Rightarrow$ (iii) : from universal algebra.

(iii) $\Rightarrow($ i $)$ : Let $\mathcal{A} \in \mathbf{H S}(\mathcal{B})$. Then there is a $C$ such that $\epsilon: \mathcal{C} \longrightarrow \mathcal{B}$ is injective and $\rho: \mathcal{C} \longrightarrow \mathcal{A}$ is surjective: 


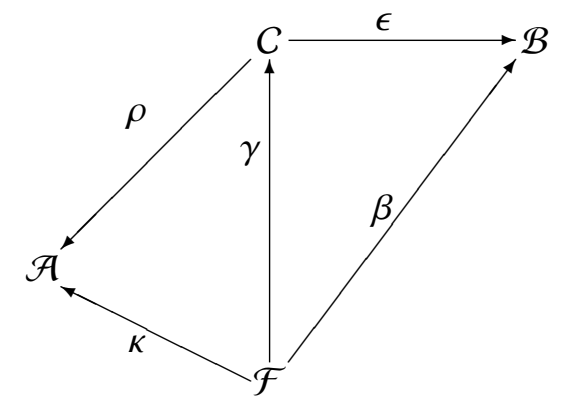

Here, $\mathcal{F}:=\mathcal{F}_{\Theta}(\operatorname{var}(\Delta))$ and $\kappa$ is the canonical mapping. $\mathcal{F}$ is free and a fortiori projective and thus $\kappa$ can be lifted over $\rho$ to $\gamma: \mathcal{F} \longrightarrow C$. Then $\beta=\epsilon \circ \gamma$. For every $\pi \in X, \kappa\left(\neg p_{c} \wedge[\pi] \Delta\right)=\kappa\left(\neg p_{c}\right)>0$ and since $\epsilon$ is injective, $\beta\left(\neg p_{c} \wedge[\pi] \Delta\right)=\epsilon \circ \gamma\left(\neg p_{c} \wedge[\pi] \Delta\right)>0$ for every $\pi \in X$. Hence $\mathcal{B}$ is extremely consistent with $\mathcal{A}$.

Lemma 16 Let $\mathcal{A}$ be s.i. and finitely presentable over $\Theta, \mathcal{B} \in \mathcal{V}(\Theta)$. If $\mathcal{B}$ weakly consistent with $\mathcal{A}$ then there is a $\mathcal{S} \in \mathbf{P}_{\mathbf{U}}(\mathcal{B})$ which is extremely consistent with $\mathcal{A}$.

Proof: Let $M$ exhaust $X$ and $\beta_{\mu}: \operatorname{var}(\Delta) \longrightarrow B$ be such that $\bar{\beta}_{\mu}\left(\neg p_{c} \wedge[\mu] \Delta\right)>0$. Then define $\mathcal{S}:=\prod \mathcal{B} / U, \sigma:=\prod \beta_{\mu} / U$, where $U$ is an ultrafilter on $M$ which contains all sets of the form $M_{\mu}:=\{\widehat{\mu}: \widehat{\mu} \supset \mu\}$. Such an ultrafilter exists because $M_{\mu} \cap M_{\widehat{\mu}} \supseteq M_{\bar{\mu}} \neq \emptyset$ for some $\bar{\mu} \supseteq \mu \cup \widehat{\mu}$. Then $\sigma\left(\neg p_{c} \wedge[\mu] \Delta\right)>0$ for every $\mu \in M$, for $\left\{\widehat{\mu}: \bar{\beta}_{\widehat{\mu}}\left(\neg p_{c} \wedge[\mu] \Delta\right)>0\right\} \supseteq\{\widehat{\mu}: \widehat{\mu} \supset \mu\}=M_{\mu} \in U$. Hence $\mathcal{S}$ is extremely consistent with $\mathcal{A}$.

This lemma can be generalized as follows: If $M$ satisfies $\forall \mu_{1}, \mu_{2} \exists \mu_{3}: \mu_{1} \cup \mu_{2} \subset \mu_{3}$ and for every $\mu \in M$ there is a $\mathcal{B}_{\mu} \in \mathbf{B}-\mathbf{B}$ a class of algebras -, which is $\mu$-consistent with $\mathcal{A}$ then there exists a $\mathcal{S} \in \mathbf{P}_{\mathbf{U}}(\mathbf{B})$ which is $M$-consistent with $\mathcal{A}$. $\mathcal{S}$ will be explicitly constructed in the proof of the splitting theorem.

Putting our results together we get the the following result which also makes use of Jónsson's lemma on congruence distributive varieties:

Theorem 17 Let $\mathcal{A}$ be s.i. and finitely presentable over $\Theta$. Then the following assertions are equivalent for every $\mathcal{B} \in \mathcal{V}(\Theta)$ :

(i) $\mathcal{B}$ is weakly consistent with $\mathcal{A}$

(ii) $\mathcal{A} \in \mathbf{S H P}_{\mathbf{U}}(\mathcal{B})$

(iii) $\mathcal{A} \in \mathbf{H S P}(\mathcal{B})$.

Proof: $($ i $) \Rightarrow$ (ii) There is a $\mathcal{S} \in \mathbf{P}_{\mathbf{U}}(\mathcal{B})$ which is extremely consistent with $\mathcal{A}$. Hence $\mathcal{A} \in$ $\mathbf{S H}(\mathcal{S}) \subseteq \mathbf{H S P}_{\mathbf{U}}(\mathcal{B})$.

(ii) $\Rightarrow(i)$ : If $\mathcal{B}$ is not weakly consistent with $\mathcal{A}$ then there is an $M$ exhausting $X$ and a $\mu \in M$ such that $\mathcal{B} \vDash[\mu] \Delta \rightarrow p_{c}$. It follows that $\mathcal{S} \vDash[\mu] \Delta \rightarrow p_{c}$ for any $\mathcal{S} \in \mathbf{P}_{\mathbf{U}}(\mathcal{B})$. Hence $\mathcal{S}$ is not extremely consistent with $\mathcal{A}$ and thus $\mathcal{A} \notin \mathbf{S H}(\mathcal{S})$. Since this valid for all $\mathcal{S} \in \mathbf{P}_{\mathbf{U}}(\mathcal{B}), \mathcal{A} \notin \mathbf{S H P}_{\mathbf{U}}(\mathcal{B})$.

(ii) $\Leftrightarrow$ (iii) : Jónsson's lemma. 


\section{D.4 The Splitting Theorem}

Theorem 18 (Splitting Theorem) Let $\mathcal{A}$ be s.i. and finitely presentable over $\Theta$. LA is prime in $\mathcal{E} \Theta$ iff there is a $\mu \in X$ such that for all $\mathcal{B} \in \mathcal{V}(\Theta)$

(†) If $\mathcal{B}$ is $\mu$-consistent with $\mathcal{A}$ then $\mathcal{B}$ is weakly consistent with $\mathcal{A}$

Proof: $(\Rightarrow)$ Let $M=X$. Assume that ( $\dagger$ ) is not satisfied for all $\mu \in M$. For every $\mu \in M$ there is a $\mathcal{B}_{\mu}$ which is $\mu$-consistent with $\mathcal{A}$ but not $\mu^{\prime}$-consistent for a $\mu^{\prime} \in M$. Hence by the preceding theorem $\mathcal{A} \notin \mathbf{H S P}\left(\mathcal{B}_{\mu}\right)$ or $L \mathcal{B}_{\mu} \nsupseteq L \mathcal{A}$. But by the generalized Lemma 16 we know that there is a $\mathcal{S} \in \mathbf{P}_{\mathbf{U}}\left(\left\{\mathcal{B}_{\mu}: \mu \in M\right\}\right)$ which is extremely consistent with $\mathcal{A}$. So $\mathcal{A} \in \mathbf{H S}(\mathcal{S})$ and therefore $\mathcal{A} \in \mathbf{H S P}\left(\left\{\mathcal{B}_{\mu}: \mu \in M\right\}\right)$, i.e. $L \mathcal{A} \supseteq \bigcap\left\langle L \mathcal{B}_{\mu}: \mu \in M\right\rangle$. Hence $L \mathcal{A}$ is not prime in $\mathcal{E} \Theta$.

$(\Leftarrow)$ Let $(\dagger)$ be fulfilled by some $M$ and $\mu \in M$ for all $\mathcal{B} \in \mathcal{V}(\Theta)$. Let $L \mathcal{A} \supseteq \bigcap\left\langle L \mathcal{B}_{i}: i \in I\right\rangle$ i.e. $\mathcal{A} \in \mathbf{H S P}\left(\left\{\mathcal{B}_{i}: i \in I\right\}\right)$. For a suitable $\mathcal{S} \in \mathbf{P}_{\mathbf{U}}\left(\left\{\mathcal{B}_{i}: i \in I\right\}\right), \mathcal{A} \in \mathbf{H S}(\mathcal{S})$. Hence $\mathcal{S}$ is extremely consistent and a fortiori $\mu$-consistent with $\mathcal{A}$. Consequently, there exists an $i \in I$ such that $\mathcal{B}_{i}$ is $\mu$-consistent with $\mathcal{A}$. By assumption, $\mathcal{B}_{i}$ is then weakly consistent with $\mathcal{A}$ and so by Theorem 17 $\mathcal{A} \in \mathbf{H S P}\left(\mathcal{B}_{i}\right)$ and thus $L \mathcal{A} \supseteq L \mathcal{B}_{i}$. Hence $L \mathcal{A}$ is prime in $\mathcal{E} \Theta$.

Corollary 19 If $\mathcal{A}$ and $\mu$ fulfill the conditions of the Splitting Theorem we have

$$
\mathcal{E} \Theta / \mathcal{A}=\Theta\left([\mu] \Delta \rightarrow p_{c}\right) .
$$

Proof: $\mathcal{B} \in \mathcal{V}(\mathcal{E} \Theta / \mathcal{A})$ iff $L \mathcal{B} \nsubseteq L \mathcal{A}$ iff $\mathcal{A} \notin \mathbf{H S P}(\mathcal{B})$ iff $\mathcal{B}$ is not weakly consistent with $\mathcal{A}$ iff $\mathcal{B}$ is $\mu$-inconsistent with $\mathcal{A}$ for some $\mu \in X$ iff $[\mu] \Delta \rightarrow p_{c} \in L \mathcal{B}$. \&

It can be shown that if $\Lambda$ splits $\mathcal{E} \Theta$ then $\mathcal{E} \Theta / \Lambda=\Theta(P)$ for some formula $P$ and $\Lambda=L \mathcal{A}$ where $\mathcal{A} \in \mathbf{H} \mathcal{F}_{\Theta}(\operatorname{var}(P))$; thus $\mathcal{A}$ is $\operatorname{card}(\operatorname{var}(P))$-generated. We conjecture that $\mathcal{A}$ is also finitely presentable so that this assumption can effectively be dropped from the Splitting Theorem.

Conjecture 20 If $\mathcal{A}$ is prime in $\mathcal{V}(\Theta)$ then $\mathcal{A}$ is finitely presentable over $\Theta$.

In addition we would get a characterization of algebras whose logics are irreducible. For if $\mathcal{A}$ is s.i. and finitely presentable over $L \mathcal{A}$ then $L \mathcal{A}$ is irreducible iff $\mathcal{A}$ is prime in $\operatorname{HSP}(\mathcal{A})$ iff $\mathcal{A}$ satisfies $(\dagger)$ within $\mathbf{H S P}(\mathcal{A})$. Now if primeness implies finite presentability we get the

Conjecture $21 \Lambda$ is irreducible iff $\Lambda=L \mathcal{A}$ for a s.i. $\mathcal{A}$ which is finitely presentable over $L \mathcal{A}$ and satisfies $(\dagger)$ of the Splitting Theorem for $\mathbf{H S P}(\mathcal{A})$.

An important specialization of Theorem 18 is

Corollary 22 If $\mathcal{X}$ has a maximal element $\mu$, every finitely presentable s.i. algebra is a splitting algebra. In particular every finite s.i. algebra is a splitting algebra. 
Proof: $\{\mu\}$ exhausts $X$.

As a consequence we get the following theorem due to Rautenberg [10]:

Corollary 23 Let $\Theta$ be weakly transitive. Then every finite s.i. $\Theta$-algebra splits $\mathcal{E} \Theta$.

This result implies among other that if $\Theta$ is weakly transitive and has f.m.p. then no infinite s.i. $\Theta-$ algebra can be finitely presentable. If the free algebra on $k$ generators is infinite it is therefore not s.i. Looking again at the counterexample for Theorem 9 we surprisingly get that the constructed algebra is not finitely presentable although it is 2-generated.

With the help of Theorem 18 a theorem of Blok [2] can be generalized. Let us say that a finite $\mathcal{X}$-algebra is cycle-free iff $\mathcal{A} \models\left[\xi^{m+1}\right] 0$ for some $m \in \omega$. It can be seen that $\mathcal{A}$ is cycle-free iff the corresponding Kripke-structure contains no cycles. ${ }^{3}$ If $\mathcal{A} \not\left[\xi^{m}\right] 0$ then $\left[\xi^{m}\right] 0$ is an opremum of $\mathcal{A}$.

Corollary 24 (Blok [2]) Let $\mathcal{A} \in \mathcal{V}_{n}$ be finite s.i. and cycle-free. Then $\mathcal{A}$ is prime in every variety containing it.

Proof: Let $\mathcal{A} \vDash\left[\xi^{m}\right] 0$. Then $\mu:=\xi^{(m)}$ satisfies ( $\dagger$ ) in Theorem 18. For if $\mathcal{B}, \beta \models\left[\xi^{(m)}\right] \Delta \wedge\left[\xi^{m}\right] 0>$ 0 then $\mathcal{B}, \beta \models\left[\xi^{(m+r)}\right] \Delta \wedge\left[\xi^{m}\right] 0>0$ because $\vdash\left[\xi^{m}\right] 0 \rightarrow\left[\xi^{m}\right]\left[\xi^{r}\right] \Delta=\left[\xi^{m+r}\right] \Delta$ for all $r \geq 0$ whence $0<\left[\xi^{(m)}\right] \Delta \wedge\left[\xi^{m}\right] 0 \leq\left[\xi^{(m)}\right] \Delta \wedge\left[\xi^{m}\right]\left[\xi^{r}\right] \Delta$ for all $r$. Since $M:=\left\{\xi^{(r)}: r \in \omega\right\}$ exhausts $\mathcal{X}(\dagger)$ is satisfied for all $\mathcal{B}$.

In general, the application of the Splitting Theorem is not straightforward. For example, the converse of Corollary 24 is also true. This can be shown with the help of the ramification technique (see Rautenberg [12]); but there seems to be no simple proof for this-let alone for the stronger result by Blok [2] that $1 \neq \Lambda=K / N$ for some set $N$ of algebras iff $N$ is a set of finite, cycle-free algebras.

\section{E An Infinite Splitting Algebra}

In this chapter I will conctruct a modal logic which has infinitely many nonfinite splitting algebras. This logic is $\Theta:=S 4 . I_{3} .2$. Here, $S 4 . I_{3}$ is the logic of $S 4$-frames of width 3 . $\Theta$ is complete (see [Fine, 1974b]) but lacks the finite model property since it has a nonfinite splitting frame.

$\Delta_{1}$
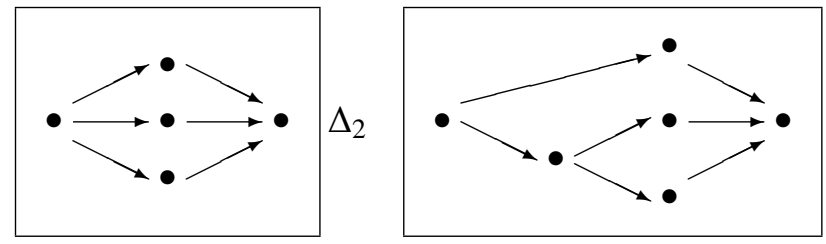

${ }^{3}$ Generally, we would define a $\pi$-cycle to be a sequence $<a_{i}: i \in n>$ such that $a_{i} \triangleleft_{\pi} a_{i+1(\bmod (n))}$ but $a_{i} \neq a_{j}$ for $i \neq j$. A cycle is then simply a $\xi$-cycle. 

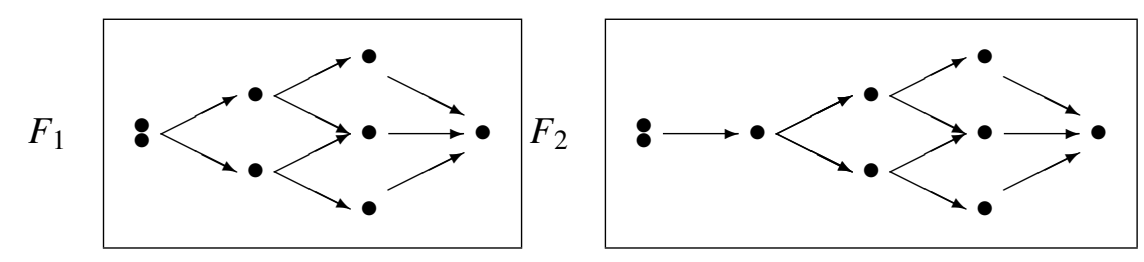

Let $\Gamma$ be the following frame

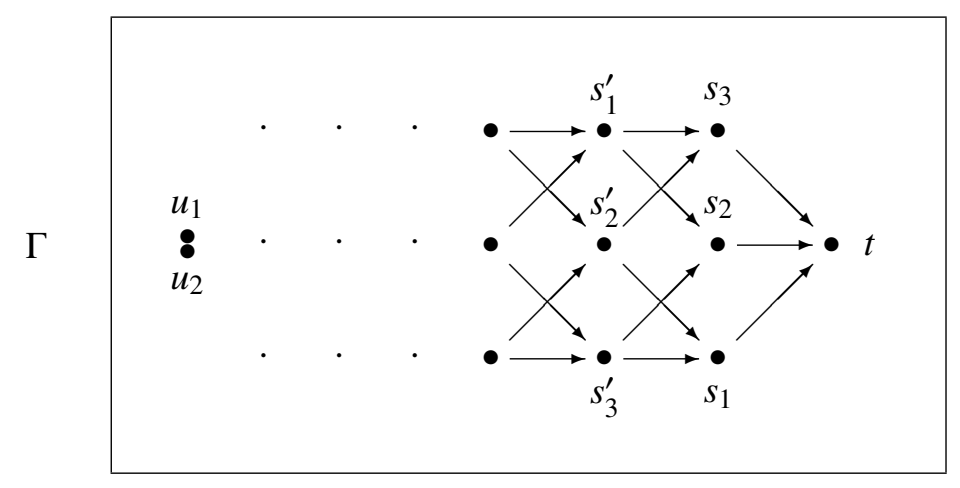

and let $\alpha$ be the following valuation on $\Gamma: \alpha\left(r_{i}\right)=\left\{u_{i}\right\}, \alpha\left(q_{i}\right)=\left\{s_{i}\right\}, \alpha(p)=\{t\}$. Also let $\mathcal{B}:=$ $\mathcal{F}_{\Theta}(X) /\langle Q\rangle$ where $X=\left\{p_{1}, p_{2}, p_{3}, q, r_{1}, r_{2}\right\}$ and

$$
\begin{aligned}
& Q=\quad p_{1} \rightarrow \neg r_{1} \wedge \neg r_{2} \wedge \neg q \wedge \neg p_{2} \wedge \neg p_{3} \\
& \wedge \quad p_{2} \rightarrow \neg r_{1} \wedge \neg r_{2} \wedge \neg q \wedge \neg p_{1} \wedge \neg p_{3} \\
& \wedge \quad p_{3} \rightarrow \neg r_{1} \wedge \neg r_{2} \wedge \neg q \wedge \neg p_{1} \wedge \neg p_{2} \\
& \wedge q \rightarrow \neg r_{1} \wedge \neg r_{2} \wedge \neg p_{1} \wedge \neg p_{2} \wedge \neg p_{3} \\
& \wedge \quad r_{1} \rightarrow \neg r_{2} \wedge \neg q \wedge \neg p_{1} \wedge \neg p_{2} \wedge \neg p_{3} \\
& \wedge \quad r_{2} \rightarrow \neg r_{1} \wedge \neg q \wedge \neg p_{1} \wedge \neg p_{2} \wedge \neg p_{3} \\
& \wedge \neg r_{1} \wedge \neg r_{2} \rightarrow \square \neg r_{1} \wedge r_{2} \\
& \wedge \quad p_{1} \rightarrow \neg \diamond r_{1} \wedge \neg \diamond r_{2} \wedge \diamond q \wedge \neg \diamond p_{2} \wedge \neg \diamond p_{3} \\
& \wedge \quad p_{2} \rightarrow \neg \diamond r_{1} \wedge \neg \diamond r_{2} \wedge \diamond q \wedge \neg \diamond p_{1} \wedge \neg \diamond p_{3} \\
& \wedge \quad p_{3} \rightarrow \neg \diamond r_{1} \wedge \neg \diamond r_{2} \wedge \diamond q \wedge \neg \diamond p_{1} \wedge \neg \diamond p_{2} \\
& \wedge q \rightarrow \square q \\
& \wedge r_{1} \rightarrow \diamond r_{2} \wedge \diamond q \wedge \diamond p_{1} \wedge \diamond p_{2} \wedge \diamond p_{3} \\
& \wedge r_{2} \rightarrow \diamond r_{1} \wedge \diamond q \wedge \diamond p_{1} \wedge \diamond p_{2} \wedge \diamond p_{3}
\end{aligned}
$$

Let $\mathcal{A}$ be the algebra of finite and cofinite subsets of $\Gamma$. I will now prove the following

Proposition 25 The canonical homomorphism $h: \mathcal{B} \longrightarrow \mathcal{A}$ induced by $h(p)=\alpha(p)$ for $p \in X$ is an isomorphism.

Having proved this proposition, the rest will easily follow. Since $\Gamma$ is generated by a single point, $\mathcal{A}$ is s.i. and since $\mathcal{A}$ is finitely presentable we have thus shown with the help of Corollary 22 that $\mathcal{A}$ is a splitting algebra of $\Theta$. 
The way we assess the proof for the proposition is by showing that the dual map for $h$ between the frames for $\mathcal{B}$ and $\mathcal{A}$ is an isomorphism. Having thus found the underlying frame of $\mathcal{B}$ it is routine to check that $\mathcal{B}$ is the algebra of finite and cofinite subsets of $\Gamma$. Before we start the proof we need a couple of definitions and observations. Let us say that a subset $N$ of a frame $\Delta$ is a slice iff it is a maximal set of incomparable points. Equivalently, since $\Delta$ is reflexive and transitive, $N$ is a slice of $h$ iff $\forall u \in h \exists m \in N: m \triangleleft u$ or $u \triangleleft m$. If $\operatorname{card}(N)=k$ we also call $N$ a k-slice. If $N$ is a slice of $h$ and $T \subseteq N$ we say that $u$ is a unifier for $T$ iff $\forall n \in N: u \triangleleft n \Leftrightarrow n \in T$ and $u$ immediately precedes every $t \in T$ but $\neg \exists n \in N: n \triangleleft u$. If $\operatorname{card}(T)=k$ we call $u$ a k-unifier. Now let $\delta: X \longrightarrow 2^{D}$ a valuation on $\Delta$.

Lemma 26 If $u_{1}$ and $u_{2}$ are within the same cluster and $h, \delta, u_{1} \vDash p$ iff $h, \delta, u_{2} \vDash p$ for all propositional variables of $X$ then $h, \delta, u_{1} \vDash P$ iff $h, \delta, u_{2} \vDash P$ for all $P$ with variables in $X$.

Lemma 27 If $u_{1}$ is a 1-unifier for $\left\{u_{2}\right\}$ and $h, \delta, u_{1} \vDash p$ iff $h, \delta, u_{2} \vDash p$ for all propositional variables of $X$ then $h, \delta, u_{1} \vDash P$ iff $h, \delta, u_{2} \vDash P$ for all $P$ with variables in $X$.

Proof: By induction on $P$. The nontrivial case is $P=\diamond Q$. Suppose, $u_{1} \vDash \diamond Q$. Then there is a $v \triangleright u_{1}$ such that $v \vDash Q$. If $v=u_{1}$ then by induction hypothesis $u_{2} \vDash Q$ whence $u_{2} \vDash \diamond Q$. If $v \neq u_{1}$ then, since $u_{1}$ immediately precedes $u_{2}, u_{2} \triangleleft v$ and thus $u_{2} \vDash \diamond Q$. Conversely, if $u_{2} \vDash \diamond Q$ then $u_{1} \vDash \diamond Q$ as well.

Lemma 28 Let $N$ be a slice and $u_{1}, u_{2}$ be unifiers for $T_{1}$ and $T_{2}$ with $T_{1} \neq T_{2}$. Then $u_{1}$ and $u_{2}$ are incomparable.

Proof: Suppose $u_{1} \triangleleft u_{2}$. Then either $u_{1}$ and $u_{2}$ are in the same cluster, in which case $T_{1} \neq T_{2}$ cannot hold, or $u_{1}$ does not immediately precede every $t \in T_{1}$.

Proof of the proposition: Let $\Delta$ be the frame of $\mathcal{B}$ whose points are the ultrafilters of $\mathcal{B}$ with $U \triangleleft T \Leftrightarrow \forall \square a \in U: a \in T$. Now take an ultrafilter $U$ such that $q \in U$. Then $\square q \in U$ by $Q$ and $\neg p_{i}, \neg r_{j}, \square \neg p_{i}, \square \neg r_{j} \in U, i \leq 3, j \leq 2$. Thus $U \triangleleft T$ iff $T=U$. Likewise, let $p_{1} \in T$. Then $\square \neg r_{1}, \square \neg r_{2}, \square \neg p_{2}, \square \neg p_{3}, \diamond q \in T$ and so $T \triangleleft T^{\prime}$ iff $T=T^{\prime}$ or $q \in T^{\prime}$ i.e. $T^{\prime}=U$. Thus one can prove that $\Delta$ contains exactly one point of depth 1 and exactly 3 points of depth 2 which form a 3 -slice. Finally, it can be shown that $r_{i} \in T$ implies $T \triangleleft T^{\prime}$ for all $T^{\prime}$. Consequently, if $S, S^{\prime}$ are two points of finite depth $>2$, we have for all generators $p \in X: p \in S \Leftrightarrow p \in S^{\prime}$. (In fact, $\forall p \in X: p \notin S)$.) Lemma 26 guarantees that all clusters with more than one point are of infinite depth.

Now we show by induction that for every $n \in \omega, n>1$, there are exactly 3 points of depth $n$ which are incomparable and that the points of depth $n+1$ form a slice of all 2-unifiers of the slice of depth $\mathrm{n}$. Looking at $\Delta$ we see that $\Delta$ is built the same way and so $\Delta \cong \Gamma$.

(A) To start the induction, we note that the points of depth 2 are a 3-slice since they satisfy $\Delta$.

(B) Now assume that the points of depth $\mathrm{n}$ are a 3-slice. Call them $x_{1}, x_{2}$ and $x_{3}$. Let $y$ be of depth $\mathrm{n}+1$. Then $y$ immediately precedes an $x_{i}$. Furthermore, $y$ must be a unifier because if it precedes a 
point of the slice it must immediately precede it. $y$ cannot be a 3-unifier because $\Delta$ omits $\Delta_{1}$, since it is a $\Theta$-frame. Also, $y$ cannot be a 1 -unifier because of Lemma 27. Thus $y$ is a 2-unifier. What is left to prove is that there are 32 -unifier for $\left\{x_{1}, x_{2}, x_{3}\right\}$. Clearly, there cannot be more. Let us therefore see whether there might be less.

(i) If there is just one unifier $y$ we can assume that it unifies $\left\{x_{1}, x_{2}\right\}$. Then $\left\{x_{3}, y\right\}$ is a 2-slice. For assume $y \rtimes u$ and $x_{3} \nless u$. Then neither $x_{1} \triangleleft u$ nor $x_{2} \triangleleft u$ can hold. Hence $u \triangleleft x_{i}$ for some $i$. If $i=3$ we are done. If $i \neq 3$ then $u$ must precede either $x_{1}$ or $x_{2}$. But then it must precede both because there is no 1-unifier for $\left\{x_{j}\right\}$. Hence $u \triangleleft y$. Since $\left\{y, x_{3}\right\}$ is a 2 -slice, and again by Lemma 27 we see that there are no 1-unifier, there can only be a single 2-unifier $z$. But then $\Delta$ does not omit $\Delta_{2}$ since the p-morhism which maps all points of depth $<n$ onto a single point maps the subframe generated by $z$ onto $\Delta_{2}$.

(ii) Thus assume that there are two 2-unifier, $y_{1}$ unifying $\left\{x_{2}, x_{3}\right\}$ and, $y_{2}$ unifying $\left\{x_{1}, x_{3}\right\}$. Again, it can be shown that now we have a 2 -slice $\left\{y_{1}, y_{2}\right\}$. Again there are no 1-unifier so there is a single 2-unifier $z$. If $z$ is contained in a 2-point cluster, the subframe generated by $z$ maps onto $F_{1}$. But if $\{z\}$ is an 1-point cluster we can show that $\{z\}$ is a 1-slice. Then the map which reduces all points between the initial cluster of $\Delta$ and $z$ onto $z$ reduces $\Delta$ to $F_{2}$.

But $\Delta$ omits all those frames. Hence the points of depth $n+1$ are a 3 -slice consisting of all the 2-unifiers of $\left\{x_{1}, x_{2}, x_{3}\right\}$.

Theorem $29 \mathcal{A}$ is a nonfinite splitting algebra of $\Theta$.

The same proof can be used not only for $\Gamma$ but for frames of similar shape. For if $\Gamma \longrightarrow \Delta$ is a subframe and $\left\{u_{1}\right\}$ is a slice of $\Delta$ such that there are only finitely many points $x \triangleleft u_{1}$ in $\Delta$ then $\Delta$ is finitely presentable and if $\Delta$ is one generated it therefore underlies a splitting algebra. Thus we have the

Theorem $30 \Theta$ has infinitely many infinite splitting algebras.

\section{References}

[Birkhoff, 1937] Garett Birkhoff. Rings of sets. Duke Mathematical Journal, 3, 1937.

[Blok, 1978] W. J. Blok. On the degree of incompleteness of modal logics. Bulletin of the Section of Logic of the Polish Academy of Sciences, 7:167 - 175, 1978.

[Blok, 1980] Wim Blok. The lattice of modal logics: An algebraic investigation. Journal of Symbolic Logic, 45:221-236, 1980.

[Fine, 1974a] Kit Fine. An ascending chain of S4 logics. Theoria, 40:110-116, 1974. 
[Fine, 1974b] Kit Fine. Logics containing K4, Part I. Journal of Symbolic Logic, 39:229-237, 1974.

[McKenzie, 1972] Ralph McKenzie. Equational bases and nonmodula lattice varieties. Transaction of the American Mathematical Society, 174:1-43, 1972.

[Rautenberg, 1977] Wolfgang Rautenberg. Der Verband der normalen verzweigten Modallogiken. Mathematische Zeitschrift, 156:123-140, 1977.

[Rautenberg, 1979] Wolfgang Rautenberg. Klassische und nichtklassische Aussagenlogik. Vieweg, Braunschweig/Wiesbaden, 1979.

[Rautenberg, 1980] Wolfgang Rautenberg. Splitting lattices of logics. Archiv für Mathematische Logik, 20:155-159, 1980.

[Whitman, 1943] Philip M. Whitman. Splittings of lattices. Transactions of the American Mathematical Society, 65:179-196, 1943. 